\title{
Carnets
}

Revue électronique d'études françaises de l'APEF

Deuxième série - 17 | 2019

Lîlle : prisme de la connaissance ou reconnaissance du monde

\section{Claude Crébillon et l'île des plaisirs}

\section{Ana Alexandra Seabra de Carvalho}

\section{(2) OpenEdition}

Journals

Édition électronique

URL : http://journals.openedition.org/carnets/10147

DOI : 10.4000/carnets. 10147

ISSN : 1646-7698

Éditeur

APEF

Référence électronique

Ana Alexandra Seabra de Carvalho, «Claude Crébillon et lîle des plaisirs », Carnets [En ligne], Deuxième série - 17 | 2019, mis en ligne le 30 novembre 2019, consulté le 10 décembre 2020. URL : http:// journals.openedition.org/carnets/10147 ; DOI : https://doi.org/10.4000/carnets.10147

Ce document a été généré automatiquement le 10 décembre 2020.

\section{(c) (7) (8)}

Carnets est mis à disposition selon les termes de la licence Creative Commons - Atribution - Pas d'utilisation commerciale 4.0 International. 


\title{
Claude Crébillon et l'île des plaisirs
}

\author{
Ana Alexandra Seabra de Carvalho
}

1 Surnommé «physicien de l'amour » ou "philosophe de la femme », Claude Crébillon (1707-1777) est avant tout un créateur livré au plaisir de l'écriture et maître d'un style incomparable qui séduit d'emblée son lecteur et l'invite à explorer en profondeur ses capacités d'interprétation. Le Sylphe (1730) est son œuvre inaugurale. Après, l'auteur publiera: Lettres de la Marquise (1732); L'Écumoire (1734); Les Égarements du coeur et de l'esprit (1736-38); Le Sopha (1742); La Nuit et le Moment (1755); Le Hasard du coin du feu (1763) ; Les Heureux Orphelins (1754); Ah Quel Conte! (1755); Lettres de La Duchesse (1768); et Lettres athéniennes (1771). Dans cette étude, nous aborderons L'Écumoire, un récit galant d'inspiration prétendument orientale, où le jeune auteur s'adonne à la problématisation du genre merveilleux et du libertinage galant, notamment dans le contexte de la séduisante Île Jonquille, ou l'île des plaisirs.

2 La charge symbolique du terme « écumoire » est ici multiple : érotique (le pouvoir de la virilité, ou son absence par la transgression de l'interdit sexuel); politico-religieux (le pouvoir royal et la question de la bulle Unigenitus ${ }^{1}$ ) et même littéraire, car on invite le lecteur à "écumer", à décanter "l'écume» de sens, les subtilités de l'écriture crébillonienne (cf. Sturm, 1976; Cazenobe, 1997 ; Sgard, 1999 et 2002; Viart, 2002 ; Labrosse, 2002). En partant du modèle du conte de fées oriental fort apprécié par son lecteur en raison de la traduction par Galland des Mille et une nuits (1704-17), Crébillon utilise cette forme littéraire à la mode tout en la transformant et en l'adaptant à son point de vue critique, notamment en ce qui concerne les questions sociopolitiques, religieuses et amoureuses, ainsi qu'à la remise en question des conventions narratives en vogue, aspect qui mérite d'être souligné dans toutes les œuvres de l'auteur. L'histoire, assez complexe d'ailleurs, est magistralement résumée par Jean Sgard :

Un conte oriental de caractère féerique déroule un cycle d'épreuves suivies de réparations. Le récit est linéaire et se déroule selon deux itinéraires inversement symétriques : le prince, frappé d'un interdit et privé de ses moyens le soir de ses noces, doit voyager et coucher avec une sorcière pour retrouver, en place de l'écumoire dont il s'est trouvé pourvu, l'organe qui lui manquait. Cependant Néadarné, privée à son tour de désirs (...), se voit contrainte de recourir à l'enchanteur Jonquille, qui lui apprendra l'amour et la rendra, apparemment vierge, à son mari. Le conte idyllique s'achève ainsi en double inconstance; le mythe de 
l'amour féerique aboutit à des compromis suspects; c'est le triomphe du "désenchantement». Sur cette toile de fond se déroule en transparence une seconde histoire, une intrigue politico-religieuse : le Prince a reçu comme tâche de faire lécher ou avaler par une vieille puis par le Grand Prêtre l'écumoire magique. Il s'y efforcera d'un bout à l'autre du récit. Le lecteur de 1734 pouvait reconnaître dans ladite écumoire une figure de la Bulle Unigenitus que Louis xiv puis le Régent, à l'instigation de Clément xi, s'étaient efforcés de faire accepter au clergé. On voit donc le Patriarche, représentant du Saint-Siège, négocier tandis que Saugrenutio, caricature des cardinaux parisiens, résiste; on voit l'aristocratie soutenir le Roi par intérêt, et le Parlement s'opposer, par tradition, à cet excès de pouvoir, et tout s'arrange à la suite de compromis peu glorieux : au terme du récit, chacun aura oublié ses convictions et léché l'écumoire, tout comme les amants auront renié leurs serments. (Sgard, $2002: 145-6)$

L'« histoire japonaise » de Tanzaï et Néadarné se déroule dans la grande Chéchianée, royaume fabuleux situé quelque part en Extrême-Orient et perdu dans la nuit des temps aussi bien que dans la mémoire des géographes (Crébillon, 1999 : 270, 275). Outre la critique sociopolitique et religieuse, comme dans toute l'œuvre de Crébillon, le thème central consiste à analyser les méandres secrets du sentiment, du désir et de la vanité qui régissent les jeux sociaux du cercle mondain de l'aristocratie parisienne. Ce contexte social particulier ressemble à une île métaphorique, un espace fermé vers l'extérieur et centré sur lui-même. Le «monde » est limité à la cour et aux salons de l'aristocratie parisienne, c'est-à-dire à la noblesse traditionnelle toute proche de la royauté. Dans l'ensemble de l'œuvre de Crébillon, ce cloisonnement sur la noblesse du sang est accentué par la préférence pour les espaces intimes en raison de la nature de son thème central, lesquels fonctionnent aussi comme des micro-îles érotiques métaphoriques : la chambre à coucher, le boudoir, le salon désert, le pavillon dans le jardin ou la petite-maison.

4 Pourtant, dans ce microcosme social l'intimité est suivie d'une exposition publique rapide et obligatoire : le récit des aventures circule avec la célérité de l'inconstance des acteurs. Alors, l'intime devient public, tout comme le public est absorbé par le cercle clos de la mondanité : l'opéra, le théâtre et le parc sont aussi des espaces fermés et restreints, autant d'îles métaphoriques où se déroulent des jeux sociaux de séduction et de pouvoir. La bonne compagnie est donc un groupe sélectif (par la naissance) et homogène (par les codes comportementaux et linguistiques). Bien sûr, ces caractéristiques ne sont pas nouvelles, car elles respectent la tradition des sociétés de cour. Cependant, par rapport au passé, la dégradation des mœurs est sans précédent. Le cérémonial et le langage jouent seuls le rôle de surveillance et d'embellissement de l'indécence. Les vrais objets de l'acuité analytique de Crébillon sont les masques rhétoriques de l'amour-goût, c'est-à-dire le langage codifié du jargon à la mode ou ton de la bonne compagnie, pratique discursive caractérisée par une déviation cynique et bienséante entre le mot et le sentiment, ainsi qu'entre le mot et l'acte. La maîtrise du code devient essentielle pour la survie dans ce monde clos, insulaire pour ainsi dire (aussi bien que pour sa préservation). La vertu n'est plus qu'une chimère, un préjugé ridicule ou un masque. En ce sens, la nouvelle civilité est complètement divorcée de l'honnêteté du XVII ${ }^{e}$ siècle et le nouvel art de plaire en société repose exclusivement sur un hédonisme de plus en plus déréglé, en modifiant l'esprit de l'héritage des siècles précédents. Alors, au XVIII ${ }^{\mathrm{e}}$ siècle, le galant homme brillant devient l'homme à bonnes fortunes, le petit-maître prétentieux, frivole et ridicule dont les talents ne servent qu'à se former une belle réputation, à savoir la «liste glorieuse " de ses conquêtes 
amoureuses, laquelle fait de lui un séducteur «à la mode». Dans Tanzaï et Néadarné, et avec une fine ironie, Crébillon dresse un inventaire détaillé des talents les plus appréciés à la Cour de la fabuleuse île Babiole, que son lecteur perçoit aisément comme le miroir parfait de la bonne compagnie de France :

C'était le plus beau danseur du monde, personne ne faisait la révérence de meilleure grâce, il devinait toutes les énigmes, jouait bien tous les jeux, tant de force que d'adresse, depuis le Trou-Madame jusques au Ballon. Sa figure était charmante, et empaquetée (...) dans les agréments les plus rares; il savait accompagner de toutes sortes d'instruments une voix charmante qu'il avait. (...) Outre les talents que je viens de nombrer (...), il faisait joliment des vers; sa conversation enjouée et sérieuse, satisfaisait également par ses grâces et sa solidité. Austère avec la Prude, libre avec la Coquette, mélancolique avec la Tendre, il n'y avait pas une Dame à la Cour dont il ne fit les délices, et pas un homme dont il ne créât la jalousie. La supériorité de son esprit ne le rendait pas insociable, complaisant avec finesse, il savait se plier à tout; il possédait mieux que personne ce langage brillant de notre île; il n'y avait personne qui ne fût comblé de l'entendre, et quoique cet être farouche intitulé le bon sens, n'agît pas toujours civilement avec ce qu'il disait, l'élégance insoutenable de ses discours faisait qu'il n'y perdait rien, ou que le bon sens, caché derrière une multitude miraculeuse de mots placés au mieux, aurait paru d'une insipidité affadissante à ses Sectateurs les plus absurdes, s'il eût été vêtu moins légèrement. (Crébillon, 1999 : 351-2)

La célébrité mondaine (la "gloire») est généralement remportée non par les faits d'armes et de politique, ni par les dons intellectuels et artistiques, mais par la galanterie, substitut du pouvoir et unique occupation d'une aristocratie oisive et futile (cf. Elias, 1985). Pour les libertins de Crébillon, plaire dans le monde signifie maitriser les ridicules en crédit: la singularité, par laquelle on se distingue des autres; la construction d'un masque de son caractère ; et la pénétration des masques d'autrui.

6 La séduction libertine devient alors une forme d'agression civilisée, qui implique la persuasion, la conquête (la défaite volontaire de la femme), l'humiliation morale suivie de l'abandon avec scandale (l'humiliation publique), pour accroître la gloire de la vanité masculine, en ajoutant une nouvelle victime à la liste (garant de son autorité et stratégie de séduction, car l'homme à la mode se fait désirer tout simplement parce qu'il est considéré comme un irrésistible objet du désir féminin). Cette forme de séduction conquiert par le langage (associé à la communication paralinguistique), par les stratégies et tactiques argumentatives et discursives et par le lexique hérité de la préciosité, langage voilé et trompeur du code ambigu, déchiffrable seulement par les initiés, car il est plein de pièges qui provoquent et déterminent les échecs des novices. Cette séduction ne cherche pas à convaincre la victime par une rationalité logique, mais plutôt à induire son adhésion par les émotions, en s'adressant à ses sentiments et vanité.

7 Ce conte de fées au parfum oriental met en évidence le séducteur merveilleux, incomparable et irrésistible, même pour la sincère, vertueuse et passionnée Néadarné. Le Génie souverain de l'île Jonquille ${ }^{2}$ est un libertin cynique, dont le désir n'est pas satisfait par la possession d'une victime trompée, mais seulement par le plaisir de la séduction persuasive, celle qui amène la victime à avouer son désir et donc à participer à sa propre défaite et humiliation. Moustache, la Fée métamorphosée en taupe pour échapper à la vengeance du Génie, le présente à Tanzaï et Néadarné comme un homme à système, qui «se fonde d'abord sur ce que les femmes à sentiments l'ont toujours trompé, en lui donnant moins de plaisir que celles qui ne se livrent à lui que par besoin, 
ou par sensualité effective; et sur la folie qu'il y a à se priver, pour un seul objet, de tous ceux qui pourraient plaire » (Crébillon, $1999: 367-8$ ).

Ce système du plaisir régnant dans l'île Jonquille scandalise les jeunes époux sincèrement amoureux l'un de l'autre. Il s'agira, en effet, d'une double initiation à l'amour et au "monde", où tous les deux sortiront de l'innocence et des préjugés juvéniles pour entrer dans le jeu social mondain des adultes et du pouvoir. Les enchantements subis par chacun d'eux, et qui les empêchent de consommer leur mariage d'amour sans l'aide de forces supérieures, signifient l'interdit et la contrainte de l'initiation érotique à la charge de la société.

Tanzaï passe d'abord son épreuve initiatique dans les bras de l'horrible Fée Concombre, souveraine de l'île des Cousins, car, selon l'oracle, la guérison du Prince (dont le membre viril s'était métamorphosé en une immense écumoire) dépend d'un voyage initiatique : "Qu'il aille; Qu'il parcoure ; // Qu'il couche; Qu'il revienne » (Crébillon, 1999 : 310). L'aventure ne commence à gagner un sens plus précis qu'avec la rencontre de la Fée au Chaudron. Adjuvante du Prince, elle le débarrasse de l'écumoire, lui révèle la destination et le but du voyage (« Vous allez dans l'île des Cousins », "Vous allez en bonne fortune », Crébillon, 1999 : 312). Enfin, elle lui offre une " ample provision » d'un élixir magique : «trente bouteilles » de la même «Eau de Santé » qu'il doit prendre la nuit de son désenchantement (Crébillon, 1999: 313). Trompé par la promesse de «bonne fortune ", Tanzaï donne libre cours à son désir imaginaire, montrant la fragilité du système du Tendre de l'union absolue et exclusive du sentiment amoureux et du désir érotique (Crébillon, 1999 : 315-6). Cependant, l'illusion et la réalité peuvent être bien différentes (Crébillon, 1999: 317-26), car la Fée Concombre est vraiment hideuse. Après la violente épreuve initiatique (à l'aide du flacon d'Eau de Santé et de l'image de Néadarné), Tanzaï, "aussi honteux que fatigué de sa bonne fortune", « reprit le chemin de Chéchian, très disposé à garder à Néadarné la fidélité la plus exacte, puisque les plaisirs dérobés lui réussissaient si mal »(Crébillon, 1999 : 326). Plus tard, il raconte cette expérience au Roi son père et à Néadarné sous la forme d'un rêve (avec toute l'ambiguïté que permet ce stratagème, Crébillon, 1999 : 337-8).

Guéri de son mal, c'est au tour de la Princesse d'être frappée par un enchantement équivalent, qui l'amène, dans la deuxième partie du roman, à partir pour l'île Jonquille à la recherche du remède que seul le Génie souverain lui peut fournir, comme le proclame l'oracle divin, cette fois "en prose, afin qu'on l'entendît mieux »: «La Princesse ne se reverra dans son premier état que le grand Génie Mange-Taupes n'en ait disposé selon sa sainte volonté » (Crébillon, 1999 : 343). Le remède annoncé effraie et la jalousie de Tanzaï et la vertu de Néadarné. Au cours de l'aventure, nous la verrons lutter continuellement entre le désir de se rétablir pour faire plaisir au Prince et la répugnance causée par le moyen imposé. Elle préfère le quiétisme amoureux à la terrible épreuve qui l'attend. Son innocence sera perdue grâce aux sophismes de Moustache et de Jonquille (même si son amour pour Tanzaï reste constant, comme il est dit à la fin (Crébillon, 1999: 436). Le système du plaisir valorise l'empire des sens au détriment du cœur, en considérant les caprices de la nature comme la source de la volupté sensuelle. Le moment où l'instinct se manifeste est imprévisible et impérieux : comme dans un rêve, la volonté de l'individu se trouve soumise au pouvoir de forces incontrôlables et profondes. La justification de l'infidélité repose sur les raisons suivantes : il ne s'agit pas d'un crime, car elle n'est pas consentie ; elle est uniquement 
due au désir de plaire au conjoint, malgré le dégoût provoqué ; c'est donc une action digne de toute son estime.

11 Tanzaï, bien que mortifié, décide de laisser partir la Princesse. Pour la rassurer, Moustache lui révèle de précieux secrets : des mots magiques pour rétablir la virginité ; le mensonge de la guérison de Tanzaï - il n'a pas rêvé, il a en fait couché avec la Fée Concombre ; et aussi que Jonquille est un Génie bien charmant (Crébillon, 1999 : 375-7). La Fée soutient encore que le sentiment est un préjugé erroné et que la nature l'éteint toujours, même si pour quelques instants seulement (Crébillon, 1999: 377-8). La nécessité pour la Princesse de passer la nuit de son désenchantement avec le Génie de l'île des plaisirs exclut l'amour (Crébillon, 1999: 379). Nonobstant, l'angoisse de Néadarné persiste au moment où elle rencontre son sacrificateur, qui l'initiera aux nouvelles règles mondaines et érotiques.

Pour Jonquille, ce n'est qu'une conquête assurée par la fatalité, mais ce séducteur apprécie les défis. Vainqueur annoncé, il ne veut pas seulement triompher de la répugnance de la jeune vierge. Son but est de la séduire, en la menant, d'un côté, à consentir au traitement ordonné par l'oracle, et de l'autre, à avouer qu'elle le désire avec ardeur. Ainsi, le maître de l'île des plaisirs, en désirant le désir de Néadarné (et non son amour), cherche à la persuader d'accepter librement son infidélité envers son mari bien-aimé et, par conséquent, les maximes du système galant (opposées aux préjugés innocents de la jeune femme amoureuse). C'est dans ce sens que Jonquille mènera la séduction, en évitant toujours, malicieusement, d'exercer la force du « coup d'autorité » et en se montrant délicat et courtois.

Les préparatifs de la séduction de la Princesse commencent bien avant son arrivée sur l'île Jonquille, présentée ici comme l'île mythique de Cythère, un locus amoenus érotique lointain et mystérieux (Crébillon, 1999 : 387 et ss.). Comme le rappelle Jean Sgard, « on peut penser au tableau de Watteau, Pèlerinage à l'île de Cythère (1717)» (Sgard, 1999 : 712). L'auteur cite alors Vivant Denon et son analyse de ce départ pour l'île de Cythère,

où l'empire de l'amour s'exerce sur tous les caractères; où la femme prude, la coquette et la sensible cèdent, chacune à sa manière, à l'entraînement général. Tout respire l'amour, l'air en est empreint, c'est lui qui enfle les voiles des bâtiments qui vont conduire les amants dans l'empire de ce despote séducteur. (apud Sgard, 1999 : 712)

D'ailleurs, héritier philosophique de l'île utopique de Thomas More (Utopia, 1516), l'espace insulaire, séparé du monde connu et isolé par l'eau, est un lieu renfermé sur lui-même, ce qui souligne son altérité. Dans la littérature française, on pourrait faire remonter à la préciosité baroque la tradition des cartographies de l'esprit et du cœur. En fait, la sentimentale "Carte du Royaume de Tendre " (Mlle de Scudéry, Clélie, 1654-60), "carte du pays et des lieux d'Amour, de ses chemins sentimentaux et traverses diverses ", lance « la mode de la géographie galante tracée et troussée par la préciosité des salons mondains » (Pelegrín, 2008 : 71). S'ensuivent : La Carte du Royaume d'Amour (Tristan L'Hermite, 1654? / 1658 posth.); «La Défense du Royaume de Coquetterie » (Abbé d'Aubignac, Lettre d'Ariste à Cléonte, 1659); Le Voyage de l'Isle d'Amour (Paul Tallemant, 1663) et ses suites; Le Pays d'Amour (Louis Moréri, 1665). On peut encore citer « La Carte du Pays de Braquerie (1668) de Bussy-Rabutin, incluse plus tard dans son Histoire amoureuse des Gaules, [qui] est une satire licencieuse de ces cartographies tendres et délicates détournées" (Pelegrín, 2008: 71). Ces récits « reposent (...) sur la métaphore géographique, les voyages, les découvertes, les îles, en particulier, emblématisent les cheminements et les égarements du cœur et de l'esprit 
[Crébillon s'en souviendra] (...) » (Pelegrín, 2008: 70). D'autre part, on pourrait tout aussi bien se rappeler du locus amoenus du conte de fées de Mme d'Aulnoy, L'île de la Félicité (1697).

Héritant de cette tradition littéraire de l'île des plaisirs, dans l'île Jonquille rien n'est laissé au hasard pour éblouir Néadarné et la prédisposer ainsi à accepter son destin, d'un esprit plus favorable : de magnifiques fêtes, banquets, chants, danses, jeux, suivis d'un opéra et du feu d'artifice, sont prévus. Délicat mais cynique, Jonquille a hâte de se rendre utile au désenchantement de la Princesse. Le séducteur dévalorise le sentiment amoureux, en affirmant le pouvoir des sens: le cœur n'est qu'une chimère ou un masque hypocrite du désir physique. En ce sens, résister est inutile, car ce n'est pas le cœur de Néadarné que Jonquille attaque, mais son désir et son innocence. D'une part, il faut désenchanter la Princesse (perte de l'innocence, des illusions et donc passage du monde enchanté de l'enfance au monde réel des adultes). D'autre part, il y a le caprice du libertin d'obtenir le consentement de la victime lors de sa chute, car c'est elle qui doit décider si elle veut que ce Génie remplisse ou pas son rôle d'initiateur. Alors, pour bien la guérir, le maître de l'île des plaisirs lui demande la nature de son mal et de raconter ses mésaventures. En apprenant quelle est la maladie de la Princesse, il l'avertit que sa guérison dépend seule de son désir de se soigner, c'est-à-dire que son «indocilité pour le remède" (Crébillon, 1999: 385) peut le rendre inefficace. Le séducteur cynique n'est pas un violeur, il tente au contraire, de persuader sa victime d'entrer volontairement dans le jeu érotique, en assumant son désir, et pas seulement par obligation sociale: «Madame, reprit Jonquille, je n'exige rien, il dépend de vous d'accepter, ou de refuser mes services. Dès ce moment, vous pouvez partir » (Crébillon, 1999 : 385).

tant, elle reste. Ensuite, le Génie cherche à l'amuser avec toutes sortes de divertissements mondains somptueux présents dans l'île des plaisirs. Pour la séduire, Jonquille tente de profiter d'un premier moment de distraction de Néadarné dans l'obscurité d'un bosquet de ses somptueux jardins insulaires, mais sans résultat (Crébillon, 1999 : 388). Avec délicatesse, le Génie décide de remettre sa conquête à plus tard. S'ensuivent le banquet, le café, le jeu, l'opéra ; et le séducteur emploie tout son art pour faire plaisir à sa victime. Néadarné est dérangée par cette tendresse excessive et, surtout, par les doutes qui commencent à l'assaillir : «devait-elle refuser ou non?» (Crébillon, 1999 : 396-7).

Touché par l'exceptionnalité de sa victime, Jonquille souhaite qu'elle tombe amoureuse de lui : « Il imaginait un plaisir extrême à chasser Tanzaï du cœur dont il était maître, et plus la victoire lui parut difficile, plus il fut flatté du triomphe » (Crébillon, 1999 : 397). Ces dispositions, ainsi que la nouvelle distraction de Néadarné pendant l'opéra, provoquent un second moment (un baiser), rapidement rejeté par la Princesse. Mais le Génie ne renonce pas à son projet, la ramenant dans un « bosquet sombre » pour mieux admirer le feu d'artifice. La vertu de Néadarné ne facilite pas la conquête, malgré la feinte passion du séducteur. Cela fait intervenir la Fée Concombre, métamorphosée en une énorme araignée, qui s'introduit sous la robe de la Princesse. La peur et l'évanouissement qui s'ensuivent concèdent au Génie le troisième moment, mis en échec cependant par le retour inopportun de la jeune femme à ses sens, déjà «à demi (...) désenchantée sans offenser sa vertu » (Crébillon, 1999 : 399-403).

En jouant le rôle de l'amant respectueux quoique désespéré, Jonquille avait déjà attendri la Princesse par ses discours timides et ses larmes feintes (Crébillon, 1999: 
401), jusqu'à ce que l'épisode de l'araignée eut changé la suite des événements. Néadarné s'en va indignée. Jonquille se montre suppliant, coupable et repentant; il annonce alors son départ et disparaît. À ce moment, Néadarné, inquiète et après avoir réfléchi au but de son voyage, le rappelle et lui promet son pardon (Crébillon, 1999: 404). Réapparaissant d'un air repentant et timide pour regagner la confiance de la Princesse, le Génie la conduit au palais. Cependant, il est déjà impatient de tant de résistance et décide de changer de stratégie, en passant à la simulation de l'indifférence pour cacher ses désirs et ainsi attaquer la vanité féminine : « Il connaissait le cœur, et il était sûr qu'en blessant la vanité de Néadarné, il l'engagerait à aller plus loin qu'elle ne voudrait»(Crébillon, 1999: 406). Mais le séducteur sait que cette "insultante froideur » doit être dosée avec précaution pour ne pas causer de l'aversion, car « s'il n'était pas sûr d'être aimé, il était certain du moins de n'être point haï. Il fallait cultiver ces heureuses dispositions, et il n'était pas encore assez bien dans le cœur de Néadarné pour pouvoir sans risque pousser loin ce manège » (Crébillon, 1999 : 407).

Sûr des effets obtenus, Jonquille retourne "avec un air animé » pour faire les compliments les plus enflammés à la beauté de la Princesse et vise à nouveau sa vanité ainsi que son cœur à travers le langage le plus passionné. Sa victoire commence à se dessiner ; Néadarné réagit déjà avec plus de douceur : "Je vous ai déjà dit que j'aimais un autre que vous, reprit-elle doucement, que voulez-vous que je vous donne?» Réponse du libertin : "Que tout ce que le Destin veut que vous me donniez (...) me soit donné par vous, et que je n'aie point la honte de le remercier d'un bonheur dont je voudrais n'avoir obligation qu'à vous seule » (Crébillon, 1999 : 408-9). Les intentions du séducteur sont claires : sa victoire doit être obtenue avec le consentement de la victime, laquelle a bien perçu la situation et accepte de considérer la question, c'est-à-dire que dans le combat intime entre désir et vertu, celle-ci commence à défaillir. Pour gagner du temps, Néadarné joue et chante ; viennent, après, le dîner et les jeux jusqu'à l'heure fatale du coucher. Toute seule avant la confrontation décisive avec le Génie, la jeune Princesse est à nouveau assaillie par les doutes les plus cruels : séduite par « le plus aimable des génies» (Crébillon, 1999: 411), sa vertu et son amour pour Tanzaï renforcent sa résistance. La naïveté initiale, qui croyait à l'amour et à la moralité idéaux, a été influencée par le système galant. Les sentiments et les sens peuvent ne pas être inséparables; la nature se montre rebelle à la volonté et à la raison; cependant le libre arbitre, qui donne la possibilité de choisir entre céder ou résister à la tentation, n'efface pas le remords, né peut-être des préjugés. Sous l'influence de ces réflexions, Néadarné décide d'empêcher l'entrée de Jonquille dans sa chambre, en verrouillant les portes. Inutile précaution, d'ailleurs, car les génies ne sont pas découragés par de tels obstacles. La sévérité de la jeune femme mène Jonquille à lui répondre avec ironie, en la piquant dans sa vanité et sa jalousie (par la référence à l'aventure de Tanzaï et Concombre) et en la menaçant de ne pas lui devenir utile dans son désenchantement (Crébillon, 1999 : 412). Il obtient ainsi l'attention de la Princesse.

20 À partir de ce moment, le Génie agira sur deux registres différents mais complémentaires : au niveau discursif et au niveau de la communication non verbale (en gérant la distance entre lui et la Princesse, en la touchant, en l'embrassant, en prenant «les plus grandes libertés » - (Crébillon, 1999: 414) -, jusqu'à ce que la guérison soit accomplie). Ainsi, tout en s'approchant de la Princesse et « en s'asseyant sur le canapé » (Crébillon, 1999 : 413), le Génie réclame sa complaisance à cause de son amour et son respect. Il se plaint ensuite de son indifférence et, « en posant doucement sa main sur la jambe de la Princesse » (Crébillon, 1999: 413), se considérant indigne 
d'une récompense, il l'avertit cependant que, dans le cas où elle regretterait à l'avenir de rester enchantée, elle devra trouver un autre guérisseur. Alors, toujours en silence, Néadarné semble commencer à céder: elle le regarde, puis détourne son regard et soupire tristement. Jonquille continue de gagner du terrain, s'avançant sur le canapé, lui prenant la main et poursuivant son discours rassurant et persuasif. Elle lui serre la main en signe de consentement, le séducteur insiste " assez indiscrètement » dans ses caresses. La " profonde rêverie » de Néadarné facilite son audace, au point qu'il réussit à l'embrasser avec ardeur, puis à prendre les plus grandes libertés. À peine sortie de sa " distraction », la Princesse lutte contre les avances du séducteur, mais en vain. Malgré sa résistance, le Génie, favorisé par son évanouissement, parvient à la désenchanter : "Jonquille, le victorieux Jonquille, loin de la secourir, goûtait à loisir les charmes de son triomphe » (Crébillon, 1999 : 414). En reprenant ses esprits, la Princesse s'indigne. Le séducteur lui dit qu'elle est libre de partir ou de rester, mais l'avertit que le désenchantement n'est efficace que s'il est volontaire. La décision de céder au désir est précipitée par l'arrivée de deux beautés demandant le remède du Génie. Alors, « elle sourit. Jonquille, content de cet aveu, l'enleva, la porta dans ce même lit où elle croyait qu'elle n'entrerait jamais, et dans l'instant, la vertu et le scrupule, bannis tous deux d'auprès d'elle, cédèrent en soupirant, leur place aux plaisirs » (Crébillon, 1999 : 416).

21 La stratégie cynique du Génie se montre efficace: la Princesse acquiesce avec un sourire. Dans ces conditions, le séducteur le prend comme une déclaration formelle et s'empresse d'obtenir les preuves, d'abord avec un "coup d'autorité » (puisqu'elle se débat toujours), puis avec des mots tendres, suivis des caresses les plus voluptueuses jusqu'à la convaincre que le sentiment n'est qu'une chimère et qu'elle doit donc se rendre à l'empire des sens. En effet, le désir conquiert la vertu et les scrupules moraux, mais pas entièrement. Néadarné n'est pas une coquette hypocrite, son dilemme intérieur est sincère, mais la nature triomphe parfois de l'amour et de la vertu. La Princesse doit avant tout se justifier pour rassurer ses scrupules : elle utilise alors le même subterfuge de Tanzaï, c'est-à-dire qu'elle cherche à voir dans l'amant l'image du Prince bien-aimé. Lorsque cette illusion échoue et que la raison gagne encore du terrain, Néadarné prétend qu'elle n'a cédé à la tentation du désir que pour l'amour de son mari. C'est également au nom des intérêts et de la vanité de Tanzaï que la Princesse décide de tester le secret de Moustache qui lui permet de rentrer intacte, mais guérie, dans les bras de son époux. Elle est satisfaite du résultat; pourtant, assaillie par le doute que le secret ne serait valable qu'une fois, malgré les promesses de la Fée, elle décide de répéter l'expérience : on voit ainsi s'affirmer, de façon subtile et malicieuse, la victoire du plaisir des sens sous le voile de l'intérêt du sentiment amoureux, c'est-àdire que l'initiation au monde des masques, plus ou moins hypocrites, est accomplie.

$\mathrm{Au}$ moment où Néadarné se prépare à partir, le Génie cherche à obtenir d'elle le consentement à de nouvelles rencontres, ce qu'elle refuse, en cherchant à se réfugier dans les arguments de toujours : l'amour de Tanzaï, le devoir, la fatalité divine qui a déterminé la nécessité de la chute initiatique, mais qui interdit de nouvelles occasions (Crébillon, 1999: 420). Cependant, cela est inutile, car le libertin Jonquille ne déviera pas de son projet de séduction cynique : après avoir obtenu les faveurs de Néadarné, il insiste pour lui faire avouer son acquiescement et son désir, en faisant d'elle la complice de sa propre séduction et, par conséquent, en lui rendant impossible de chercher refuge dans les excuses qu'elle invoque. Alors, il lui montre qui est le maître :

Quand je vous ai demandé cette permission, Princesse, reprit-il, c'est parce que

jusques au bout j'ai voulu vous devoir tous mes plaisirs. Si vous connaissiez bien ma 
puissance, vous ne douteriez pas que, malgré tous vos refus, je ne pusse vous voir quand je le voudrais, et obtenir même de votre tendresse toutes les faveurs que vous réservez à Tanzaï. Maître de prendre sa figure, c'est sous ses traits que vous me verrez et vous ne saurez jamais si c'est à lui ou à moi que vous livrerez votre cœur. (Crébillon, 1999 : 420)

Vaincue physiquement et moralement pour avoir joué le jeu de la séduction de son plein gré, Néadarné voit son tourment se perpétuer avec la dernière menace du Génie du plaisir: sous le masque de l'amour (et/ou de l'hypocrisie), il se trouve toujours l'exigence du désir sensuel. Les sens dominent l'être même en amour; il est donc inutile d'essayer de résister à son pouvoir. Ces mots, terriblement prophétiques, sont immédiatement confirmés par la nouvelle attaque du séducteur. Néadarné ne résiste plus, mue maintenant par le désir de satisfaire la demande de Moustache et d'obtenir la liberté de Cormoran. La malice de ce prétendu prétexte est accentuée par le fait que la Princesse a recouru de nouveau au secret de la Fée pour, plus facilement, «provoquer le Génie au sommeil » (Crébillon, 1999 : 420). Quand elle l'obtient, elle décide de partir ; cependant, la beauté de l'aimable Génie et le souvenir des moments de plaisir passés en sa compagnie sur l'île Jonquille la laissent troublée et « avec une peine dont elle sentit murmurer sa vertu »(Crébillon, $1999:$ 421). Son aventure prouve que la nature trouble le sentiment, car Jonquille est passé du sacrificateur craint et détesté au " plus aimable des génies » et à son libérateur : «Ce n'était pas qu'elle l'aimât, mais elle n'avait rien à lui imputer de ce qui s'était passé entre eux, et ne pouvait raisonnablement le regarder que comme son libérateur » (Crébillon, 1999: 423). Bien que son amour pour Tanzaï n'ait pas changé, elle sait maintenant que le cœur et les sens sont indépendants, mais elle doit également conserver sa "gloire » et sa "tranquillité » (Crébillon, 1999: 431) afin de s'assurer de l'amour et de l'estime du Prince (ainsi que de la société). De cette manière, elle se trouve dans la nécessité de mentir : elle raconte son aventure comme un rêve et se sert du secret de Moustache :

Je vous ai déjà dit, Prince, (...) que je ne sais si Concombre n'est plus à craindre pour nous ; j'ai cependant lieu de soupçonner que sa colère ne pourra plus troubler nos jours.

Jonquille, ennuyé de ma résistance, après avoir tenté auprès de moi tout ce que l'amour peut suggérer de séductions, me laissa enfin à moi-même. Je fus conduite dans un appartement dont je fermai toutes les portes sur moi : couchée sur un canapé, j'y déplorais ma situation ; je me mis à rêver profondément à mes malheurs, je m'endormis, et après le songe le plus funeste pour ma pudeur et mon amour, songe qui, tout éveillée que je suis, me remplit de terreur et de honte, je crus m'apercevoir d'un changement considérable... (Crébillon, 1999 : 430)

Il s'agit en vérité d'une petite revanche sur l'aventure prétendument onirique de Tanzaï : «Punis tous deux de la même manière, pourquoi ne nous aurait-on pas donné le même remède? » (Crébillon, 1999 : 430).

En conclusion, la morale de l'histoire, selon la perspective de Claude Crébillon, est que dans un monde dominé par la galanterie, celui de «la bonne compagnie » du xviiie siècle représenté ici surtout par le microcosme particulier de l'île Jonquille, la survie dépend de l'acceptation des conventions sociales. En ce sens, un mariage d'amour comme celui de Tanzaï et de Néadarné implique, selon Colette Cazenobe, « un dosage subtil de sage dissimulation et de nécessaire mauvaise foi », car «pour vivre heureux ensemble, il ne faut exiger l'impeccabilité ni de l'autre ni de soi. C'est encore de l'amour, mais c'est aussi un compromis » (Cazenobe, 1991:123-4). Alors, le jeu féerique des métamorphoses physiques et morales que les jeunes époux éprouvent dans ce conte 
galant au parfum oriental sert à amener le lecteur français de l'époque à réfléchir aux codes de conduite tant dans l'intimité que dans la société et la politique. Il sert également à interroger les relations complexes, chères à l'auteur, entre le cœur, l'esprit et les sens. En tant que conteur, Crébillon retrouve et transforme avec beaucoup d'imagination un vaste fond culturel où se croisent les différentes traditions occidentales et orientales associées au merveilleux, en particulier dans le long épisode de l'île Jonquille. Un tel travail de transformation fait partie d'un processus esthétique et littéraire qui problématise l'exotisme merveilleux dans la littérature du xvIII siècle. Cependant, la féerie fournit à l'auteur moraliste un moyen ludique et transgressif pour critiquer la morale et les mœurs, révéler l'hédonisme et le culte de la volupté de son temps, ainsi que les manœuvres du pouvoir politique.

\section{BIBLIOGRAPHIE}

CARVAlHo, Ana Alexandra Seabra de (2003). o Jogo do desejo em Claude Crébillon. Estudo dos processos retóricos da sedução. Lisboa : Fundação Calouste Gulbenkian/FCT.

CAZENOBE, Colette (1991). Le système du libertinage de Crébillon à Laclos. SVEC nº 282. Oxford : The Voltaire Foundation.

CAZENOBE, Colette (1997). Crébillon fils ou la politique dans le boudoir. Paris-Genève : Éd. Honoré Champion.

CRÉBILLON, Claude (1999). « Tanzaï et Néadarné, histoire japonaise », in Jean Sgard (org.). Cuuvres complètes de Claude Crébillon. Paris : Classiques Garnier Multimédia, t. I, pp. 269-439 (éd. critique du texte par J. Sgard).

ELIAS, Norbert (1985). La Société de Cour. Paris : Flammarion.

LABROSSE, Claude (2002). «Crébillon, les fées et les signes », in Régine Jomand-Baudry et JeanFrançois Perrin (orgs.). Le conte merveilleux au XVIII siècle : Une poétique expérimentale. Paris : Éditions Kimé, pp. 102-112.

PELEGRÍN, Benito (2008). D’un temps d'incertitude. Cabris : Éditions Sulliver.

SGARD, Jean (1999). «Introduction », in Claude Crébillon (1999). « Tanzaï et Néadarné, histoire japonaise », in Jean Sgard (org.). CEuvres complètes de Claude Crébillon. Paris : Classiques Garnier Multimédia, t. I, pp. 237-268.

SGARD, Jean (2002). « Allégorie d'une écumoire », in Régine Jomand-Baudry et Jean-François Perrin (orgs.). Le conte merveilleux au XVIII ${ }^{e}$ siècle : Une poétique expérimentale. Paris : Éditions Kimé, pp. 140-148.

STURM, Ernest (éd.) (1976). « Introduction », in Crébillon fils. L'Écumoire ou Tanzaï et Néadarné, histoire japonaise. Paris : A. G. Nizet, pp. 9-95.

VIART, Thierry (2002). «L'Écumoire de Claude Crébillon : le conte dévoyé ou les délices de l'île Babiole ", in Régine Jomand-Baudry et Jean-François Perrin (orgs.). Le conte merveilleux au XVIII siècle: Une poétique expérimentale. Paris : Éditions Kimé, pp. 400-408. 


\section{NOTES}

1. La bulle Unigénitus est « l'objet de la crise religieuse et parlementaire qui mobilise l'opinion française dans les années $1730 »($ Sturm, 1976 : 13). Le décret de la Constitution Unigenitus avait été promulgué en 1713 et enregistré au Parlement de Paris en 1714, sur l'ordre de Louis XIV. À la mort du roi, la bulle devient un sujet de discorde nationale (cf. Sgard, 1999 : 701). Cette bulle, « par laquelle Louis XIV avait cru pouvoir venir à bout du jansénisme, a été extorquée au pape Clément XIII en septembre 1731. Sur son sens, personne ne s'entend, mais il faut obéir; l'arbitraire royal est flagrant. Le haut clergé va se soumettre assez vite; ce sera le cas en particulier du cardinal de Rohan » (Sgard, 1999 : 248). Selon Sgard, « le jugement de Crébillon, qui suppose un certain recul par rapport à la promulgation de la bulle, est très lucide; la querelle de la Constitution a provoqué dans le clergé le schisme qu'elle prétendait éviter, et divisé l'Église jusqu'à la fin du siècle » (Sgard, 1999 : 718).

2. On trouve ici quatre îles féériques : l'île Métissao, où règne l'altière Princesse Roussa Blaffarda, qui veut se marier au Prince Tanzaï; l'île des Cousins, dominée par la vieille Fée Concombre (nourrice et conseillère de Roussa Blaffarda); l'île Babiole de la Fée Barbacela (protectrice de Tanzaï et Néadarné. Cette fée satirise « le cardinal Fleury, qui préside aux destinées du royaume ", Sturm, 1976: 296-7); et l'île Jonquille, l'île des plaisirs, située au milieu d'un grand lac, domaine d'« un Génie puissant, proche parent de Concombre (...) ; il est d'un tempérament fort amoureux, et l'île Jonquille, où il fait sa demeure ordinaire, n'est qu'un sérail composé des plus belles personnes de l'univers »(Crébillon, 1999 : 343-4).

\section{RÉSUMÉS}

Connu à son époque comme "physicien de l'amour» ou "philosophe de la femme ", Claude Crébillon (1707-1777) est un auteur livré au plaisir de l'écriture et maître d'un style incomparable qui séduit d'emblée son lecteur et l'invite à des jeux complexes d'interprétation. Dans cette étude, nous aborderons L'Écumoire, un récit galant d'inspiration prétendument orientale, dans lequel le jeune auteur s'adonne à la problématisation du genre merveilleux et du libertinage galant, notamment en profitant du contexte de la séduisante île Jonquille.

Known in his time as "physicist of love " or "philosopher of women ", Claude Crébillon (1707-1777) is an author surrendered to the pleasure of writing and master of an incomparable style that seduced his reader and invites him to complex interpretation games. In this study, we will approach L'Écumoire, a gallant tale of supposedly oriental inspiration, in which the young author devotes himself to the problematization of the marvellous genre and gallant libertinism, namely in making the most of the context of the seductive île Jonquille.

\section{INDEX}

Mots-clés : Crébillon (Claude), séduction, île des plaisirs

Keywords : Crébillon (Claude), seduction, pleasure island 
AUTEUR

ANA ALEXANDRA SEABRA DE CARVALHO

Universidade do Algarve | CLEPUL

aacarva[at]ualg.pt 